\title{
Spatial transcriptomics: Gene expression in space, time and numbers
}

Eukaryotic gene expression is an array of complex processes that must fine-tune with cellular needs to maintain homeostasis, yet flexible enough to respond to external cues and signals. Transcriptomics is the average output of cellular gene expression wherein messenger RNA copy the information inscribed in the DNA to instruct protein synthesis by the cellular ribosomes in the cytoplasm. So far, the knowledge gained from the transcriptomic-based studies was similar to the Heisenberg uncertainty dilemma. Information about these transcripts could be quantitated with utmost accuracy at the cost of losing information about their cellular spatial coordinates. In other words, we may quantitate the transcripts by PCR or the modernday next-generation sequencing but cannot comment on where these transcripts originated or are located in the cells/tissues. Suppose we, however, accurately try to localize these transcripts using in-situ hybridization-like techniques; in that case, we are uncertain about its actual copy numbers due to the semi-quantitative nature of these methods. Its genuinely a Heisenbergian tradeoff that baffled the biologist for a long time until now; an innovative solution seems to be in place. Spatial transcriptomics (ST) is the perfect marriage between localization and quantitation without compromising either. It is a method that allows simultaneous visualization and quantitative analysis of the transcriptome by performing histological sections on glass slides incubated with oligonucleotides containing positional barcodes to generate high-quality cDNA libraries, which may be used for quantitation by RNA-sequencing. ${ }^{1}$ Increasingly, the scientific community seems to realize this technology's full potential, ${ }^{2}$ as evidenced by the sheer number of publications in the past 2 years. This technology is the first of its kind to provide an unbiased whole transcriptome analysis with anatomical information from tissue sections where these transcripts are expressed. A time laspse serial longitudinal ST is powerful to identify the temporal transcript oscillations and decay at unprecedented accuracy. ${ }^{3}$ Cells of different types are spatially and structurally organized within the tissue matrix to perform their complex functions. Uncovering the complex spatial architecture of heterogenous tissue is crucial for our understanding of the disease's pathology. ${ }^{4}$ Understanding the disease is the primary step towards

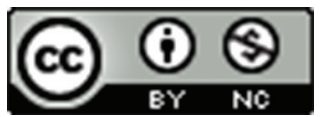

This work is licensed under a Creative Commons Attribution-NonCommercial 4.0 International License.

its remedy, and ST is a powerful tool to address this with precision. $^{.}$

Ruby Dhar', Arun Kumar'2, Subhradip Karmakar ${ }^{3}$

${ }^{1}$ Scientist, ${ }^{3}$ Associate-Professor, Department of Biochemistry, All India Institute of Medical Sciences, New Delhi, ${ }^{2}$ Professor, Department of Biochemistry, Jagannath Gupta Institute of Medical Sciences and Hospital, Kolkata, West Bengal, India

Address for Correspondence:

Dr. Arun Kumar, Professor, Department of Biochemistry, Jagannath Gupta Institute of Medical Sciences and Hospital, Kolkata, West Bengal, India. Mobile: +91-7584089886. E-mail: arun732003@gmail.com Dr. Subhradip Karmakar, Additional Professor, Department of Biochemistry, All India Institute of Medica Sciences, New Delhi, India. Mobile: +91-9868445358.

E-mail: subhradipaiims@gmail.com

\section{REFERENCES}

1. Moor AE and Itzkovitz S. Spatial transcriptomics: Paving the way for tissue-level systems biology. Curr Opin Biotechnol. 2017;46:126-133.

https://doi.org/10.1016/j.copbio.2017.02.004

2. Song $Q$ and Su J. DSTG: Deconvoluting spatial transcriptomics data through graph-based artificial intelligence. Brief Bioinform. 2021;22(5):bbaa414.

https://doi.org/10.1093/bib/bbaa414

3. Ståhl PL, Salmén F, Vickovic S, Lundmark A, Navarro JF, Magnusson $\mathrm{J}$, et al. Visualization and analysis of gene expression in tissue sections by spatial transcriptomics. Science. 
2016;353(6294):78-82.

https://doi.org/10.1126/science.aaf2403

4. Burgess DJ. Spatial transcriptomics coming of age. Nat Rev Genet. 2019;20(6):317.

https://doi.org/10.1038/s41576-019-0129-z
5. Vickovic S, Eraslan G, Salmén F, Klughammer J, Stenbeck L, Schapiro D, et al. High-definition spatial transcriptomics for in situ tissue profiling. Nat Methods. 2019;16(10):987-990.

https://doi.org/10.1038/s41592-019-0548-y

Authors Contribution:

All authors contributed equally towards scripting of this editorial.

Work attributed to:

Jagannath Gupta Institute of Medical Sciences, Budge Budge, Kolkata- 700137

All India Institute of Medical Sciences, New Delhi-110029

ORCID ID:

Dr. Ruby Dhar - (D) https://orcid.org/0000-0003-3600-6554

Dr. Arun Kumar - (1) https://orcid.org/0000-0002-8800-0296

Dr. Subhradip Karmakar - (10) https://orcid.org/0000-0002-4757-8729

Source of Funding: None, Conflicts of Interest: None. 